\title{
ПОНЯТИЕ ОБОБЩЕННОГО МОДУЛЯ И ЕГО ПРИМЕНЕНИЕ К ТЕОРИИ КВАЗИКОНФОРМНЫХ ОТОБРАЖЕНИЙ
}

\author{
в. В. КРИвов
}

Математический факультет Московского Государственного Университета SU 117234 Москва B-234, CCCP

В теории квазиконформньгх отображений очень широко используется понятие модуля семейства кривых или семейства поверхностей, а также связанное $c$ ним понятие конформной емкости. С помощью этих понятий решаются как качественшые задачи теории, так и задачи, связанные с отысканием в некотором классе отображения, наиболее близкого к конформному. В этой заметке с помошью вводимого автором понятия обобщенного модуля решаются некоторые задачи второго из указанньг типов, то есть так называемые экстремальные задачи теории. При этом мы ограничиваемся рассмотрением диффеоморфизмов, хотя дифференциальные свойства рассматриваемых отображений в ряде случаев без изменения формулировок окончательньх результатов могут быть более общими.

\section{1. Дифференциальные формы и нормы}

Пусть $M$ - ориентируемое риманово многообразие размерности $n, T_{x}$ касательное пространство к $M$ в точке $x \in M$, а $\Lambda_{n}^{l}\left(T_{x}\right)$ - пространство антисимметрических полилинейньт функщий порядка $l \leqslant n$, определенных на декартовом произведении $T_{x} \times T_{x} \times \ldots \times T_{x}$, состоящем из $l$ сомножителей.

Выбирая в каждой точке некоторый элемент $\omega(x) \in \Lambda_{n}^{l}\left(T_{x}\right)$, получим отображение $\omega: x \rightarrow \omega(x)$, задающее на $M$ дифференщиальную форму порядка $l$.

Выбирая в $T_{x}$ ортонормированный базис $\left(e_{1}, \ldots, e_{n}\right)$, обозначим $\left(d x_{1}, \ldots\right.$ $\left.\ldots, d x_{n}\right)$ дуальный базис сопряженного пространства $T_{x}^{*}$. Тогда

$$
\omega(x)=\sum_{i_{1} \ldots i_{l}} \omega_{i_{1} \ldots i_{l}} d x_{i_{1}} \wedge \ldots \wedge d x_{i_{l}},
$$

где символ $\wedge$ обозначает внешнее умножение, а суммирование производится по всем наборам различных индексов, таких что $1 \leqslant i_{1}<i_{2}<\ldots$ $\ldots<i_{l} \leqslant n$ 
Положим

$$
|\omega(x)|=\sqrt{\sum_{i_{1} \ldots i_{l}} \omega_{i_{1} \ldots i_{l}}^{2}} .
$$

Если $x_{1}, \ldots, x_{n}$ - локальные координаты в некоторой окресности точки $x$, то выражение (1.1) можно рассматривать тоже в этой окресности, хотя (1.2) в окресности справедливо лишь для ортогональньт координат.

Покажем, что значение $|\omega(x)|$ не зависит от выбора ортонормированного базиса в $T_{x}$. Пусть $\left(\tilde{e}_{1}, \ldots, \tilde{e}_{n}\right)$ - другой ортонормированный базис в $T_{x}$, a $\left(d y_{1}, \ldots, d y_{n}\right)$ - дуальный к нему базис в $T_{x}^{*}$. Тогда

$$
d x_{i}=\sum_{j=1}^{n} a_{i j} d y_{j},
$$

где $\left(a_{i j}\right), i, j=1, \ldots, n$ - ортогональная матрица. Обозначим через $A_{i_{1}}^{k_{1} \ldots k_{l}}$ минор этой матрицы, образованный элементами, стоящими в строках с номерами $i_{1}, \ldots, i_{l}$ и столбцах с номерами $k_{1}, \ldots, k_{l}$, причем $1 \leqslant k_{1}<\ldots$ $\ldots<k_{l} \leqslant n$. Будем иметь

Поэтому

$$
d x_{i_{1}} \wedge \ldots \wedge d x_{i_{l}}=\sum_{k_{1} \ldots k_{l}} A_{i_{1} \ldots i_{l}}^{k_{1} \ldots k_{l}} d y_{k_{1}} \wedge \ldots \wedge d y_{k_{l}} .
$$

$$
\sum_{i_{1} \ldots i_{l}} \omega_{i_{1} \ldots i_{l}} d x_{i_{1}} \wedge \ldots \wedge d x_{i_{l}}=\sum_{k_{1} \ldots k_{l}} \tilde{\omega}_{k_{1} \ldots k_{l}} d y_{k_{1}} \wedge \ldots \wedge d y_{k_{l}},
$$

где положено

$$
\tilde{\omega}_{k_{1} \ldots k_{l}}=\sum_{i_{1} \ldots i_{l}} \omega_{i_{1} \ldots i_{l}} A_{i_{1} \ldots i_{l}}^{k_{1} \ldots k_{l}}
$$

Отсюда

$$
\begin{aligned}
\sum_{k_{1} \ldots k_{l}} \tilde{\omega}_{k_{1} \ldots k_{l}}^{2}=\sum_{i_{1} \ldots i_{l}} \omega_{i_{1} \ldots i_{l}}^{2} \sum_{k_{1} \ldots k_{l}}\left(A_{i_{1} \ldots i_{l}}^{k_{1} \ldots k_{l}}\right)^{2}+ \\
+\sum_{\substack{i_{1} \ldots i_{l} \\
j_{1} \ldots j_{l}}}^{\prime} 2 \omega_{i_{1} \ldots i_{l}} \omega_{j_{1} \ldots j_{l}}\left(\sum_{k_{1} \ldots k_{l}} A_{i_{1} \ldots i_{l}}^{k_{1} \ldots k_{l}} A_{j_{1} \ldots j_{l}}^{k_{1} \ldots k_{l}}\right),
\end{aligned}
$$

где штрих над знаком суммы означает, что суммирование производится по всем различным наборам индексов $i_{1}, \ldots, i_{l}$ и $j_{1}, \ldots, j_{l}$, то есть таким наборам, у которьх различаются хотя бы по одному из входящих в них индексов.

Из формулы Бине-Коши (см. [1]) и ортогональности матрищы $\left(a_{i j}\right)$ вытеқает, что

$$
\sum_{k_{1} \ldots k_{l}}\left(A_{i_{1} \ldots i_{l}}^{k_{1} \ldots k_{l}}\right)^{2}=1 ; \quad \sum_{k_{1} \ldots k_{l}} A_{i_{1} \ldots i_{l}}^{k_{1} \ldots k_{l}} A_{j_{1} \ldots j_{l}}^{k_{1} \ldots k_{l}}=0,
$$

где $\left(i_{1}, \ldots, i_{l}\right)=\left(j_{1}, \ldots, j_{l}\right)$. Следовательно

$$
\sum_{k_{1} \ldots k_{I}} \tilde{\omega}_{k_{1} \ldots k_{l}}^{2}=\sum_{i_{1} \ldots i_{l}} \omega_{i_{1} \ldots i_{l}}^{2}
$$

и независимость величины $|\omega(x)|$ от выбора ортогонального нормированного базиса в $T_{x}$ установлена.

Запишем $\omega(x)$ для фиксированного $x$ в виде (1.1) и введем дифференциальную форму $\omega^{*}$ порядка $k-l$, полагая

$$
\omega^{*}(x)=\sum_{i_{1} \ldots i_{l}} \omega_{i_{1} \ldots i_{l}} \sigma\left(i_{1}, \ldots, i_{l}, j_{1}, \ldots, j_{m}\right) d x_{j_{1}} \wedge \ldots \wedge d x_{j_{m}},
$$

где в каждом слагаемом индексы $j_{1}, \ldots, j_{m}$ - это все те числа из набора $(1, \ldots, n)$, которьх нет среди чисел $\left(i_{1}, \ldots, i_{l}\right)$, причем $1 \leqslant j_{1}<\ldots<j_{m} \leqslant n$, $m=n-l$, а $\sigma\left(i_{1}, \ldots, i_{l}, j_{1}, \ldots, j_{m}\right)$ - сигнатура перестановки $\left(i_{1}, \ldots, i_{l}\right.$, $\left.j_{1}, \ldots, j_{m}\right)$.

Поскольку многообразие ориентируемо, то (1.6) задает $\omega^{*}$ на всем многообразик. На самом деле, $\omega^{*}$ не зависит от выбора ортонормированного базиса (той же ориентации) в $T_{x}$, а при изменения ориентации базиса лишь меняет знак.

Из (1.1) и (1.6), обозначая через $d x=d x_{1} \wedge \ldots \wedge d x_{n}$ элемент объема в пространстве $T_{x}$, а затем рассматривая дифференциальную форму $d v(x)=$ $=d x$, полугшим

$$
\omega(x) \wedge \omega^{*}(x)=|\omega(x)|^{2} d x .
$$

Нетрудно проверить, что величина $|\omega(x)|$, определенная равенством (1.2), обладает всеми свойствами нормы в пространстве $\Lambda_{n}^{l}\left(T_{x}\right)$ и что операция * линейна и $|\omega(x)|=\left|\omega^{*}(x)\right|$, то есть задает изоморфизм пространств $A_{n}^{l}\left(T_{x}\right)$ и $\Lambda_{n}^{n-l}\left(T_{x}\right)$ с сохранением нормы.

Если $L$ - некоторое ориентируемое подмногообразие размерности $l$, содержащееся в $M$, функция $\omega$ - измерима и $|\omega|$ интегрируема на $L$, то справедливо неравенство

$$
\left|\int_{L} \omega\right| \leqslant \int_{L}|\omega| d v
$$

Для доказательства этого неравенства достаточно рассмотреть параметризацию некоторой окрестности в $L$, воспользоваться неравенством КошиБуняковского и разбиением единицы. Мы опускаем здесь это доказательство.

Обозначим через $L_{p}(M ; l)$, где $p \geqslant 1-$ фиксированное число, совокупность всех дифференщиальных форм порядка $l(l \leqslant n)$, определенньх на многообразии $M$, имекоших измеримые коэффициенты, и таких, что функция $|\omega(x)|^{p}$ интегрируема на $M$. Условимся при этом отождествлять две формы, если они различағотся лишь на множестве меры нуль.

Введем в $L_{p}(M ; l)$ норму, полагая

$$
\|\omega\|=\left(\int_{M}|\omega(x)|^{p} d v\right)^{1 / p} .
$$

Убедимся в том, что это действительно норма. Ограничимся проверкой не- 
равенства треугольника, так как другие свойства нормы, очевидно, вьтолняются. Пользуясь неравенством Минковсқого, получим

$$
\begin{aligned}
\left\|\omega_{1}+\omega_{2}\right\| & =\left(\int_{M}\left|\left(\omega_{1}+\omega_{2}\right)(x)\right|^{p} d v\right)^{1 / p} \leqslant\left(\int_{M}\left(\left|\omega_{1}(x)\right|+\left|\omega_{2}(x)\right|\right)^{p} d v\right)^{1 / p} \leqslant \\
& \leqslant\left(\int_{M}\left|\omega_{1}(x)\right|^{p} d v\right)^{1 / p}+\left(\left.\int_{M}\left|\omega_{2}(x)\right|^{p} d v\right|^{1 / p}=\left\|\omega_{1}\right\|+\left\|\omega_{2}\right\| .\right.
\end{aligned}
$$

Итак, $L_{p}(M ; l)$ - полное нормированное пространство. Так как значения входящих в него функций лежкат в $\Lambda_{n}^{l}\left(T_{x}\right)$, которое является нормированным пространством размерности $n ! / l !(n-l) !$, то все результаты, относящиеся к пространствам векторнозначньх интегрируемьг функций, сохранлются.

Справедливы, в частности, неравенства Кларқсона (см. [5]):

(a) $\quad 2\left(\left\|\omega_{1}\right\|^{p}+\left\|\omega_{2}\right\|^{p}\right) \leqslant\left\|\omega_{1}+\omega_{2}\right\|^{p}+\left\|\omega_{1}-\omega_{2}\right\|^{p} \leqslant$

$$
\begin{aligned}
& \text { (b) } 2\left(\left\|\omega_{1}\right\|^{p}+\left\|\omega_{2}\right\|^{p}\right)^{q-1} \leqslant\left\|\omega_{1}+\omega_{2}\right\|^{q}+\left\|\omega_{1}-\omega_{2}\right\|^{q} \text {, } \\
& \text { (c) }\left\|\omega_{1}+\omega_{2}\right\|^{p}+\left\|\omega_{1}-\omega_{2}\right\|^{p} \leqslant 2\left(\left\|\omega_{1}\right\|^{q}+\left\|\omega_{2}\right\|^{q}\right)^{p-1} \text {, }
\end{aligned}
$$

где $p \geqslant 2, q=p /(p-1)$, а если $1<p \leqslant 2$, то эти неравенства меняются на противоположные.

\section{2. Обобщенный модуль и экстремальная форма}

Пусть $F$ - некоторое подмножество пространства $L_{p}(M ; l)$. Велкчина

$$
\mu(F ; M)=\inf _{\omega \in F}\|\omega\|
$$

назытается обобщенным модулем многообразия $M$ по совокупности (см. также работу автора [8]).

Если для некоторой $\omega_{0} \in F \mu(F ; M)=\left\|\omega_{0}\right\|$, то форма $\omega_{0}$ называется экстремальной.

Творема 2.1. Eсли множсество $F$ замкнуто в $L_{p}(M, l),(p>1)$ и выпукло, то экстремальная дифференчиальная форма для него суиеетвует и единственна.

Доказательство. Пусть вначале $p \geqslant 2$. По определенжю нижней грани существует последовательность $\omega_{v} \in F(v=1,2, \ldots)$, такая что

$$
\mu(F ; M)=\lim _{\nu \rightarrow \infty}\left\|\omega_{\nu}\right\| .
$$

Фиксируя $\varepsilon>0$, найдем номер $\boldsymbol{v}_{0}$, такой что для всех $\boldsymbol{v}>\boldsymbol{v}_{0}$ вьтолняется неравенство

$$
\left\|\omega_{\nu}\right\|^{p} \leqslant \mu^{p}(F ; M)+(\varepsilon / 2)^{p} .
$$

Тогда для любьх двух номеров $\nu_{1}, \nu_{2}>v_{0}$ в силу выпуклости $F$ имеем: $\left(\omega_{v_{1}}+\omega_{v_{2}}\right) / 2 \in F$ и, следовательно,

$$
\mu^{p}(F ; M) \leqslant\left\|\frac{\omega_{\nu_{1}}+\omega_{\nu_{2}}}{2}\right\|^{p} .
$$

Вместе с неравенством Кларксона (1.10) (а) и (2.3) это дает:

$$
\begin{aligned}
\mu^{p}(F ; M)+\left\|\frac{\omega_{\eta_{1}}-\omega_{\eta_{2}}}{2}\right\|^{p} & \leqslant 2^{p-1}\left(\left\|\frac{\omega_{v_{1}}}{2}\right\|^{p}+\left\|\frac{\omega_{v_{2}}}{2}\right\|^{p}\right) \leqslant \\
& \leqslant 2^{p-1} \cdot 2^{-p+1}\left(\mu^{p}(F ; M)+(\varepsilon / 2)^{p}\right)=\mu^{p}(F ; M)+(\varepsilon / 2)^{p},
\end{aligned}
$$

$$
\left\|\omega_{y_{1}}-\omega_{v_{2}}\right\|<\varepsilon
$$

Последовательность $\omega_{v}$ оказывается фундаментальной, а поскольку пространство $L_{p}(M ; l)$ полное, то существует предел $\omega_{0}=\lim _{p \rightarrow \infty} \omega_{p}$. Теперь из

(2.2) и непрерывности нормы вытекает, что

$$
\mu(F ; M)=\left\|\omega_{0}\right\|,
$$

и форма $\omega_{0}$ оказывается экстремальной. Ее единственность следует из (2.5). В самом деле, если $\omega_{\nu_{1}}$ и $\omega_{\nu_{2}}$ в этом неравенстве считать экстремальными, то $\varepsilon$ в этом случае, как это видно из рассуждения, можно считать произвольным, и, значит, эти две формы совпадакот. При $1<p \leqslant 2$ рассуждение то же самое, но использует (1.10)(c).

Теорема 2.2. Предположсим, ито совокунность $F$ вместе с любой дифференциальной формой $\omega \in F$ содержит дифференчиальную форму $\omega+\varphi$, где $\varphi-$ произвольная точная форна с компактным носителем в $M$, а экстремальнал форма $\omega_{0}$ непрерьвно дифберениируема. Тогда $\omega_{0}$ удовлетворяет уравнению

$$
d\left(\left|\omega_{0}\right|^{p-2} \omega_{0}^{*}\right)=0
$$

где $d$ - внешний дифференциал.

Доказательство. Пусть $\psi$ - произвольная непрерывно-дифференцируемая форма порядка $l-1$, носитель которой содержится в достаточно малом открытом шаре $B \subset M$ и пусть $\varphi=d \psi$. Функция $g(t)=\int_{M}\left|\omega_{0}+t \varphi\right|^{p} d v$ достигет минимума при $t=0$. Поэтому с учетом (1.7)

$$
\begin{aligned}
g^{\prime}(0) & =\left(\frac{d}{d t} \int_{M}\left|\omega_{0}+t \varphi\right|^{p} d v\right)_{t=0}= \\
& =p\left[\int_{M}\left(\left|\omega_{0}+t \varphi\right|^{2}\right)^{p / 2-1} \frac{d}{d t}\left(\left|\omega_{0}+t \varphi\right|^{2} d v\right)\right]_{t=0}=p \int_{M}\left|\omega_{0}\right|^{p-2} \varphi \wedge \omega_{0}^{*}=0 .
\end{aligned}
$$

Обозначим для краткости $\omega_{1}=\left|\omega_{0}\right|^{p-2} \omega_{0}^{*}$ и проинтегрируем по $B$ равенство $d\left(\omega_{1} \wedge \psi\right)=d \omega_{1} \wedge \psi+(-1)^{m} \omega_{1} \wedge d \psi$. Применяя формулу Стокса, получим

$$
0=\int_{\partial B} \omega_{1} \wedge \psi=\int_{B} d\left(\omega_{1} \wedge \psi\right)=\int_{B} d \omega_{1} \wedge \psi+(-1)^{m} \int_{B} \omega_{1} \wedge \psi .
$$

Как видно из (2.8), второе слагаемое здесь обращается в нуль. Значит, равно нулю и первое слагаемое, откуда в силу произвольности $\psi$ и вытекает (2.7). 
Рассмотрим 2 примера.

1. Пусть $M$ - кольцевал область в $n$-мерном евклидовом пространстве $R^{n}$, то есть такая область, дополнение которой до $R^{n}$ содержит ровно две связные компоненты $C_{0}$ и $C_{1}$, а совокупность $F$ состоит из всех точных дифференциальньгх форм $\omega$ первого порядка на $M$, таких что $\int_{\gamma} \omega=I$ для любого пути, соединяющего $C_{0}$ и $C_{1}$ в $M$. Тогда $[\mu(F ; M)]^{p}$ есть модуль порядка $p$ семейства всех таких путей или (при $p=n$ ) конформная емкость области $M$ по Левнеру (см. [9]), $\omega$ можно задать в виде векторного поля $\nabla u$, где функция $u$ непрерьвна в $R^{n},\left.u\right|_{c_{0}}=0,\left.u\right|_{C_{1}}=1$, пнтеграл в (2.1) представляет собой интеграл Дирихле, а уравнение (2.7) принимает вид:

$$
\text { (2.10) } \operatorname{div}\left(|\nabla u|^{p-2} \nabla u\right)=0 .
$$

В частности при $p=2$ получается уравнение Лапласа, а при $p=n$ известное уравнение для экстремальной функции қонформной емкости. Этот пример оправдывает название введенного понятия.

2. Вычислим модуль прямого произведения $M=M_{1} \times M_{2}$ двух римановьхх многообразий размерностей $l$ и $m$, соответственно, $l+m=n$, имеющих конечные объемы $v_{i}=\int_{M_{i}} d v_{i}, i=1,2$, причем метрику в $M$ зададим формулой $d s^{2}=d s_{1}^{2}+d s_{2}^{2}$, где $d s_{i}$ - метрика в $M_{i}$. Предположим, что $M_{1}$ имеет гладқий край $\partial M_{1}$, а совокупность $F=F\left(M_{1}\right)$ состоит из всех непрерывныг на $\left(M_{1} \cup\right.$ $\left.\cup \partial M_{1}\right) \times M_{2}$, замкнутых в $M$ дифференциальных форм порядка $l$, удовлетворяющцх условиям:

1. $\int_{M_{1} \times x_{2}} \omega \geqslant 1$ для некоторой точки $x_{2} \in M_{2}$ при фиксированной ориентация $M_{1} \times x_{2}$

2. $\omega\left(T_{x}^{l}\left(\partial M_{1} \times M_{2}\right)\right)=0$ для всех $x \in \partial M_{1} \times M_{2}$.

Пусть $\tilde{x}_{2} \in M_{2}$ и $\gamma$ - гладкий путь, соединяющий $x_{2}$ иг $\tilde{x}_{2}$ в $M_{2}$. Тогда $M_{1} \times \gamma$ есть сингулярная $(l+1)$-мерная цепь в $M_{1} \times M_{2}$. Пользуясь формулой Стокса при соответствующем выборе ориентации в $M_{1} \times \tilde{x}_{2}$ и учитывая условие 2, которому удовлетворяет совокупность $F$, получим:

(2.11) $0=\int_{M_{1} \times \gamma} d \omega=\int_{\partial\left(M_{1} \times \gamma\right)} \omega=\int_{\partial M_{1} \times \gamma} \omega+\int_{M_{1} \times \tilde{x}_{2}} \omega-\int_{M_{2} \times x_{2}} \omega=\int_{M_{1} \times \tilde{x}_{2}} \omega-\int_{M_{2} \times x_{2}} \omega$, так что $\left|\int_{M_{1} \times x_{2}} \omega\right| \geqslant 1$ для всех $x_{2} \in M_{2}$ и $\omega \in F\left(M_{1}\right)$.

Возьмем в (1.8) в качестве $L$ многообразие $M_{1} \times x_{2}$ и проинтегрируем это неравенство по $M$. Для $\omega \in F\left(M_{1}\right)$ после применения теоремы Фубини, получим:

$$
v_{2} \leqslant \int_{M_{2}} d x_{2}\left|\int_{M_{1} \times x_{2}} \omega\right| \leqslant \int_{M_{2}} d x_{2} \int_{M_{1} \times x_{2}}|\omega| d x_{1}=\int_{M_{1} \times M_{2}}|\omega| d v .
$$

Отскода, пользуясь неравенством Гелюдера, найдем:

$$
v_{2} \leqslant \int_{M}|\omega| d v \leqslant\left(\int_{M}|\omega|^{p} d v\right)^{1 / p}\left(\int_{M} d v\right)^{(p-1) / p}
$$

и, следовательно, поскольку $v=v_{1} \cdot v_{2}$

$$
\|\omega\| \geqslant v_{2}^{1 / p} / v_{1}^{(p-1) / p} .
$$

Рассмотрим теперь на $M_{1}$ дифференциальную форму $\sigma_{1}=d v_{1}$, задағоцую объем. Так как ее порядок равен $l$ и совпадает с размерностью многообразия $M_{1}$, то $d \sigma_{1}=0$. Положим $p\left(x_{1}, x_{2}\right)=x_{1}$, где $x_{1} \in M_{1}, x_{2} \in M_{2}$. Отображение $p$ проектирует $M$ на $M_{1}$. Фиксируя ориентацию $M_{1}$, будем считать, что при этом проектировании $M_{1} \times x_{2}$ на $M_{1}$ ориентапия сохраняется.

Возъмем теперь произвольную точку $x \in M$ и векторы $\bar{a}_{1}, \ldots, \bar{a}_{l}$ в $T_{x}$. Зададим на $M$ дифференциальную форму $\omega_{0}=\frac{1}{v_{1}} p^{*} \sigma_{1}$, полагая

$$
p^{*} \sigma_{1}(x)\left(\bar{a}_{1}, \ldots, \bar{a}_{l}\right)=\sigma_{1}\left(p^{\prime}\left(\bar{a}_{1}\right), \ldots, p^{\prime}\left(\bar{a}_{l}\right)\right),
$$

где $p^{\prime}(x)$ - производная $p$ в точке $x$. Легко видеть, что:

$$
d \omega_{0}=\frac{1}{v_{1}} d p^{*}\left(\sigma_{1}\right)=\frac{1}{v_{1}} p^{*}\left(d \sigma_{1}\right)=0,
$$

так что дифференщиальная форма $\omega_{0}$ замкнута в $M$. Проверим, что $\omega_{0} \in$ $\in F\left(M_{1}\right)$. Поскольку $p$ осуществляет взаимно-однозначное отображение $M_{1} \times x_{2}$ на $M_{1}$ (если фиксировать $x_{2}$ ), то

$$
\int_{M_{1} \times x_{2}} \omega_{0}=\int_{M_{1} \times x_{2}} \frac{1}{v_{1}} p^{*}\left(\sigma_{1}\right)=\int_{p\left(M_{1} \times x_{2}\right)} \frac{1}{v_{1}} \sigma_{1}=1 .
$$

Кроме того, заметим, что касательное пространство к $\partial M_{1}$ имеет в каждой точке $x$ размерность $l-1$, в силу чего для любых $l$ векторов $\bar{b}_{1}, \ldots, \bar{b}_{l} \in$ $\in T_{x}\left(\partial M_{1}\right)$ справедливо равенство $\sigma_{1}\left(b_{1}, \ldots, b_{l}\right)=0$. Отсюда, если $\left(\bar{a}_{1}, \ldots\right.$ $\left.\ldots, \bar{a}_{l}\right) \in T_{x}^{l}\left(\partial M_{1} \times M_{2}\right)$, то

$$
\begin{aligned}
\omega_{0}(x)\left(\bar{a}_{1}, \ldots, \bar{a}_{l}\right) & =\frac{1}{v_{1}} p_{1}^{*}\left(\sigma_{1}\right)\left(\bar{a}_{1}, \ldots, \bar{a}_{l}\right)= \\
& =\frac{1}{v_{1}} \sigma_{1}\left(p^{\prime}\left(\bar{a}_{1}\right), \ldots, p^{\prime}\left(\bar{a}_{l}\right)\right)=0,
\end{aligned}
$$

то есть оба условия вытолнены.

Если $\quad x=\left(x_{1}^{(1)}, \ldots, x_{1}^{(l)}, x_{2}^{(1)}, \ldots, x_{2}^{(m)}\right) \in M, \quad$ где $\quad x_{1}^{(1)}, \ldots, x_{1}^{(l)}$ - ортогональные координаты в $T_{x_{1}}\left(M_{1}\right)$, а $x_{2}^{(1)}, \ldots, x_{2}^{(m)}$ - ортогональные координаты в $T_{x_{2}}\left(M_{2}\right)$, то $\sigma_{1}=d x_{1}^{(1)} \wedge \ldots \wedge d x_{2}^{(l)}$ и

$$
\omega_{0}=\frac{1}{v_{1}} p^{*}\left(d x_{1}^{(1)} \wedge \ldots \wedge d x_{1}^{(l)}\right)=\frac{1}{v_{1}} d x_{1}^{(1)} \wedge \ldots \wedge d x_{1}^{(l)}
$$

откуда $\left|\omega_{0}(x)\right|=1 / v_{1}$ для всех $x \in M$. По теореме Фубини теперь получим:

$$
\left\|\omega_{0}\right\|=\left(\int_{M}\left|\omega_{0}\right|^{p} d v\right)^{1 / p}=\left(\int_{M_{2}} d x_{2} \int_{M_{1} \times x_{2}} v_{1}^{-p} d v\right)^{1 / p}=v_{2}^{1 / p} / v_{1}^{(p-1) / p}
$$


Вместе с (2.12) это и дает искомую величину модуля

$$
\mu\left(F\left(M_{1}\right) ; M_{1} \times M_{2}\right)=v_{2}^{1 / p} / v_{1}^{(p-1) / p},
$$

причем формула, задающая $\omega_{0}$, эффективно описывает экстремальную дифференциальную форму .

\section{3. Квазиинвариантность обобщениого модуля}

Пусть $f: M \rightarrow N$ - дифференцируемое отображение многообразия $M$ в другое риманово многообразие $N$, размерность которого равна $\nu$, к, вообще говоря не совпадает с размерностью $M$. Для любой точки $x \in M$ возникает линейное отображение $f^{\prime}(x): T_{x} \rightarrow T_{f(x)}$, где $T_{f(x)}$ - касательное пространство к $N$ в точке $f(x)$. Это линейное отображкение естественным образом индуцирует линейное отображение $f_{x}^{*}: \Lambda_{\nu}^{l}\left(T_{f(x)}\right) \rightarrow \Lambda_{n}^{l}\left(T_{x}\right)$, сопоставляющее форме $\omega$ порядка $l$ на $N$ форму $f^{*} \omega$ порядка $l$ на $M$ (сравни (2.16)). Положким $y=f(x)$.

Фиқсируя $y$, введем норму $\left|f_{*}^{*}\right|$ по формуле:

$$
\left|f_{y}^{*}\right|=\sup _{|\omega(y)| \neq 0}\left|f^{*} \omega(y)\right| /|\omega(y)| .
$$

Например, пусть размерности $M$ и $N$ совпадают, $f^{\prime}(x)$ имеет в надлежащим образом выбранных ортонормированньгх базисах вид $d y_{i}=\lambda_{i} d x_{i}$, $i=1,2, \ldots, n, \lambda_{1} \geqslant \lambda_{2} \geqslant \ldots \geqslant \lambda_{n}$.

Тогда из (1.2) и (3.1) для любого $x \in M$ получим:

$$
\left|f^{*} \omega(y)\right| /|\omega(y)|=\sqrt{\sum_{i_{1} \ldots i_{l}}\left(\omega_{i_{1} \ldots i_{l}} \lambda_{i_{1}} \ldots \lambda_{i_{l}}\right)^{2}} / \sqrt{\sum_{i_{1} \ldots i_{l}} \omega_{i_{1} \ldots i_{l}}^{2}} \leqslant \lambda_{1} \cdot \ldots \cdot \lambda_{l},
$$

так что

$$
\text { (3.2) } \quad\left|f_{y}^{*}\right| \leqslant \lambda_{1} \cdot \ldots \cdot \lambda_{l} \text {. }
$$

Если же взять $\omega(y)=d y_{1} \wedge \ldots \wedge d y_{l}$, то убедимся, что на самом деле в (3.2) стоит равенство, то есть

$$
\left|f_{y}^{*}\right|=\lambda_{1} \cdot \ldots \cdot \lambda_{l}
$$

Полезно заметить также, что $\left|f^{*} \omega(y)\right| \leqslant\left|f_{y}^{*}\right| \cdot|\omega(y)|$. Величина

$$
K_{l}(f)=\underset{x \in M}{\operatorname{essup}} \frac{\left(\lambda_{1} \cdot \ldots \cdot \lambda_{n}\right)^{l / n}}{\lambda_{n-1+1} \cdot \ldots \cdot \lambda_{n}}
$$

называется $l$-ой дилатаиией дифференцируемого отображения $f$. Если $\lambda_{1}=\lambda_{2}=\ldots=\lambda_{n}$ в каждой точке $x \in M$, то в правой части (3.4) получится 1. В этом случае отображение конформно

Отметим еще, что

(3.5) $\quad K_{1}(f)=\underset{x \in M}{\operatorname{esssup}} \frac{\left(\lambda_{1} \cdot \ldots \cdot \lambda_{n}\right)^{1 / n}}{\lambda_{n}} ; \quad K_{n-1}(f)=\underset{x \in M}{\operatorname{esssup}} \frac{\lambda_{1}}{\left(\lambda_{1} \cdot \ldots \cdot \lambda_{n}\right)^{1 / n}}$;

так что $K_{1}(f)$ есть внутренняя дилатачия, а $K_{n-1}(f)$ - внешняя дилатация отображения $f$ (по терминологии работы [2]).
Творема 3.1. Если $f: M \rightarrow f(M)$ диффеоморфизм, $F$ - некоторая совокупность дифференчиальных форм порядка $l$ в $f(M), f^{*} F-$ совокупность всех дифференчиальных форм в $M$, полученных из форм совокупности $F$ с помощью $f^{*}$, то для обобщенного модуля при $p=n / l$ имеют место неравенства

$$
\frac{1}{K_{l}(f)} \mu(F ; f(M)) \leqslant \mu\left(f^{*} F ; M\right) \leqslant K_{n-l}(f) \mu(F ; f(M)) .
$$

Доказательство. Поскольку для почти всех $x \in M$ имеем неравенство $\left|f_{y}^{*}\right| /\left|\operatorname{det} f^{\prime}(x)\right|^{l / n} \leqslant K_{n-l}(f)$, то для любой $\omega \in F$ :

$$
\begin{aligned}
\left(\int_{f(M)}|\omega(y)|^{n / l} d y\right)^{l / n} & \geqslant\left(\int_{M} \frac{\left|f^{*} \omega(y)\right|^{n / l}}{\left|f_{y}^{*}\right|^{n / l}}\left|\operatorname{det} f^{\prime}(x)\right| d x\right)^{l / n} \geqslant \\
& \geqslant \frac{1}{K_{n-l}(f)}|| f^{*} \omega \| \geqslant \frac{1}{K_{n-l}(f)} \mu\left(f^{*} F ; M\right) .
\end{aligned}
$$

Отсюда следует правое из неравенств (3.6). Левое неравенство получается тем же способом при рассмотрении обратного диффеоморфизма.

Следствив. Если отображсение $f$ конформно, то

$$
\mu\left(f^{*} F ; M\right)=\mu(F ; f(M)),
$$

то есть обобценный модуль при $p=n / l$ является конформным инвариантом. Это сразу вытекает из (3.6), так как для конформного отображения $K_{l}(f)=$ $=K_{n-1}(f)=1$.

Рассмотрим 2 примера применения формулы (3.8).

1. Пусть $M$ - область в $R^{3}$, заданная условиями

$$
x_{1}^{2}+x_{2}^{2}<\alpha x_{3}^{2} ; \quad x_{3}>0 ; \quad 0<a<|x|<b
$$

где $x=\left(x_{1}, x_{2}, \ldots, x_{3}\right)$ декартовы прямоугольные координаты, $\alpha$ - положительное число.

Обозначим $p: x \rightarrow x /|x|$ проекцию пространства $R^{3}$ на сферу $S^{2}$ радиуса 1 и пусть $y_{1} \in R$, а $y_{2} \in S^{2}$.

Тогда $f:\left(x_{1}, x_{2}, x_{3}\right) \rightarrow(\ln |x| ; x /|x|)=\left(y_{1}, y_{2}\right)$ есть отображение множества $M$ в декартово произведение $R \times S^{2}$, причем $f(M)$ будет декартовым произведением интервала $(\ln a, \ln b)$ на сферическую шапочку $Q_{\alpha}^{\prime}=\{x \in$ $\left.\in S^{2}: x_{1}^{2}+x_{2}^{2}<\alpha x_{3}^{2} ; x_{3}>0\right\}$.

В любой точке $x \in M$ :

$$
\lambda_{1}=(\ln |x|)^{\prime}=1 /|x| ; \quad \lambda_{2}=\lambda_{3}=1 /|x|,
$$

так что отображение $f$-конформное. Из (2.19) и (3.8), полагая $p=n / l$, где $l=1, n=3$, получим

$$
\mu\left(f^{*} F ; M\right)=v_{2}^{1 / 3} / v_{1}^{2 / 3} .
$$

13 Banach Center t. 11 
Но $v_{1}=\ln b-\ln a=\ln \left(\frac{b}{a}\right)$, а $v_{2}$ представляет собой площадь сферической шапочки $Q_{\alpha}$, так что

$$
v_{2}=2 \pi(\sqrt{1+\alpha}-1) / \sqrt{1+\alpha} .
$$

Совокупность $f^{*} F$, где $F$ описана в примере 2 раздела 2, состоит в этом случае из всех непрерывных на $M$ вместе с частью границы $\{x \in \partial M ;|x|=a$ или $|x|=b\}$ дифференциальных форм первого порядка вида $f^{*} \omega$, где $\omega \in F$. Так как $\omega$ - замкнута, то $f^{*} \omega$ тоже замкжута и, в силу односвязности области $M$, существует первообразная, то есть в нашем случае функция $u$, такая что $f^{*} \omega=d u ; u=$ const при $|x|=a$ и $|x|=b$, и можно положить $\left.u\right|_{|x|=a}=0,\left.u\right|_{|x|=b}=1$. Уравнение (2.7) принимает вид (2.10), а из (3.9) и (3.10) получается величина модуля

$$
\mu\left(f^{*} F ; M\right)=\left(2 \pi(\sqrt{1+\alpha}-1) / \sqrt{1+\alpha} \ln ^{2} \frac{b}{a}\right)^{1 / 3}
$$

Это, естественно (после возведения в степень 3), совпадает с величиной конформной емкости по Лёвнеру (см. [2] и [9]), а также с величиной модуля семейства всех кривых, соединяющихх $B_{0}=\{x \in \partial M ;|x|=a\}$ и $B_{1}=$ $=\{x \in \partial M ;|x|=b\}$ в $M$.

2. Пусть $M$ - тороидальная область в $R^{3}$, полученная врашением круга $\left(x_{2}-a\right)^{2}+x_{3}^{2}<1, a>1$, расположендого в плоскости $\left(x_{2}, x_{3}\right)$ вокруг оси $O x_{3}$. Полуплоскость $\left\{\left(x_{1}, x_{2}, x_{3}\right) \in R^{3} ; x_{1}=0 ; x_{2}>0\right\}$ можно рассматривать как плоскость Лобачевского, вводя в ней метрику $d s / x_{2}$, где $d s$ - обычная евклидова метрика. Упомянутый круг будет кругом $B$ в этой плоскости, его неевклидов радиус равен $\frac{1}{2} \ln \frac{a+1}{a-1}$, неевклидова длина огружности $l=2 \pi / \sqrt{a^{2}-1}$, а неевклидова площадь

$$
v_{2}=2 \pi /\left(a+\sqrt{a^{2}-1}\right) \sqrt{a^{2}-1} .
$$

Обозначим $h:\left(x_{1} ; x_{2} ; x_{3}\right) \rightarrow\left(0 ; \sqrt{x_{1}^{2}+x_{2}^{2}} ; x_{3}\right)=y_{2}$ отображение пространства с выброшенной осью $О x_{3}$ на упомянутую выше плоскость Лобачевского, через $y_{2}$ будем обозначать точку этой плоскости, а через $y_{1}$ точку на окружности $S^{1}$ радиуса 1 в плоскости $\left(x_{2}, x_{3}\right)$, заданной уравненшем $x_{1}^{2}+x_{2}^{2}=1$.

Тогда

$$
f:\left(x_{1} ; x_{2} ; x_{3}\right) \rightarrow\left(\frac{x_{1}}{\sqrt{x_{1}^{2}+x_{2}^{2}}} ; \frac{x_{2}}{\sqrt{x_{1}^{2}+x_{2}^{2}}} ; h(x)\right)=\left(y_{1}, y_{2}\right)
$$

определяет отображение области $M$ на декартово произведение $S^{1} \times B$, причем в любой фиксированной точке $x \in M$

$$
\lambda_{1}=\frac{1}{\sqrt{x_{1}^{2}+x_{2}^{2}}} ; \quad \lambda_{2}=\lambda_{3}=\frac{1}{\sqrt{x_{1}^{2}+x_{2}^{2}}},
$$

так что отображение $f$ конформное. Из (2.19) и (3.8), полагая $p=n / l, l=1$, $n=3$, получим (3.9), но на этот раз $v_{1}=2 \pi ; v_{2}$ задается формулой (3.12). Совокупность $f^{*} F$ состоит из всех непрерывных на $M$ замкнутьх дифференциальньгх форм 1-го порядка, таких что $\int_{\gamma} \omega=1$ для любой окружности, гомотопной окружности: $x_{1}^{2}+x_{2}^{2}=a^{2} ; x_{3}=0$.

Поэтому величина модуля оказывается равной

$$
\mu\left(f^{*} F ; M\right)=\left[2 \pi\left(a+\sqrt{a^{2}-1}\right) \sqrt{a^{2}-1}\right]^{-1 / 3}
$$

а после возведения в куб совпадает с модулем семейства всех кривьг, гомотопных упомянутой окружнтости.

Тем же способом можно подсчитать модули многих других областей, сводя их к случаю прямого произведения.

\section{4. Структура экстремальных отображений}

Пусть римановы многообразия $M_{1}$ и $N_{1}$ имект размерность $l$, римановы многообразия $M_{2}$ и $N_{2}$ имегот размерность $m$, причем $l+m=n$, и пусть $M_{1}$ имеет гладкий край $\partial M_{1}$, а $N_{1}$ имеет гладкий край $\partial N_{1}$.

Рассмотрим прямое произведение $M=M_{1} \times M_{2}$ и обозначим через $F\left(M_{1}\right)$ совокупность дифференциальньх форм, описанную в примере 2 раздела 2, причем $p=n / l$. Аналогично определим $F\left(N_{1}\right)$.

Пусть теперь $\mathfrak{M}$ - совокупность диффеоморфизмов $\left(M_{1} \cup \partial M_{1}\right) \times M_{2}$ на $\left(N_{1} \cup \partial N_{1}\right) \times N_{2}$, обладағощих свойствами:

1. Существуют $x_{2} \in M_{2}, y_{2} \in N_{2}$ и гладкое ориентируемое. многообразие $Q$ размерности $l+1$ в $N_{1} \times N_{2}$ такие, что

$$
\partial Q=f\left(M_{1} \times x_{2}\right)-N_{1} \times y_{2}+\Sigma,
$$

где $\Sigma \subset \partial N_{1} \times N_{2}$.

2. Для любого $f \in \mathfrak{M}: f\left(\partial M_{1} \times M_{2}\right)=\partial N_{1} \times N_{2}$. В частности, если $\partial N_{1}=\varnothing$, то свойство 1 означает, что $f\left(M_{1} \times x_{2}\right)$ и $N_{1} \times y_{2}$ гомологичны B $N_{1} \times N_{2}$.

Сохраняя для $v_{i}$ тот же смысл, что и в (2.19), обозначим через $\tilde{v}_{i}$ объем $N_{i}(i=1,2)$.

Теорема 4.1. Предположии, что суиествутот диффеоморфизмы $g_{1}: M_{1} \cup$ $\cup \partial M_{1} \rightarrow N_{1} \cup \partial N_{1}$ и $g_{2}: M_{2} \rightarrow N_{2}$ с постоянньии якобианами $I_{1}$ и $I_{2}$, соответственно, $a v_{i} u \tilde{v}_{i}$ отличны от $0 u \infty$. Тогда для любого диффеоморфизма $f \in \mathfrak{M}$ справедливо неравенство:

$$
K_{n-l}(f) \geqslant\left(I_{1}^{m} / I_{2}^{l}\right)^{1 /(l+m)},
$$

причем знак равенства можст достигаться лииь для диффеоморфизмов, обладаюицх свойствами (в обозначениях раздела 3):

(a) $\left|f_{y}^{*}\right| /\left|\operatorname{det} f^{\prime}(x)\right|^{l / n}=K_{n-l}(f)=$ const; 
(b) если $\omega_{0}$-экстремальная форма в $N$, то $f^{*} \omega_{0}$-экстремальная форма в $M$,

(c) $\left|f^{*} \omega_{0}(y)\right|=\left|f_{y}^{*}\right| \cdot\left|\omega_{0}(y)\right|$ для всех $y \in N$.

Доказательстьо. Вначале заметим, что имеет место включение $f^{*} F\left(N_{1}\right) \subset$ $\subset F\left(M_{1}\right)$. Действительно, для любой $\omega \in F\left(N_{1}\right)$ выберем $x_{2}$ и $y_{2}$ так, чтобы имело место (4.1). Применяя формулу Стокса и учитывая, что $\int_{\Sigma} \omega=0$, получим

$$
\int_{M_{1} \times x_{2}} f^{*} \omega=\int_{f\left(M_{1} \times x_{2}\right)} \omega=\int_{N_{1} \times y_{2}} \omega \geqslant 1 .
$$

Если еще учесть 2-е свойство отображения $f$ и 2-е условие для $f^{*} \omega$, то получим $f^{*} \omega \in F\left(M_{1}\right)$, то есть справедливость упомянутого включения. Теперь из определения модуля и (2.19) при $p=n / l$ получаем: (4.3) $\quad \mu\left(f^{*} F\left(N_{1}\right) ; M_{1} \times M_{2}\right) \geqslant \mu\left(F\left(M_{1}\right) ; M_{1} \times M_{2}\right)=v_{2}^{l / n} / v_{1}^{m / n}$.

Kроме того,

$$
\mu\left(F\left(N_{1}\right) ; N_{1} \times N_{2}\right)=\tilde{v}_{2}^{l / n} / \tilde{v}_{1}^{m / n}
$$

откуда, пользуясь правым неравенством из (3.6), найдем:

$$
\begin{aligned}
K_{n-l}(f) & \geqslant \frac{\mu\left(f^{*} F\left(N_{1}\right) ; M_{1} \times M_{2}\right)}{\mu\left(F\left(N_{1}\right) ; N_{1} \times N_{2}\right)} \geqslant \frac{\mu\left(F\left(M_{1}\right) ; M_{1} \times M_{2}\right)}{\mu\left(F\left(N_{1}\right) ; N_{1} \times N_{2}\right)}= \\
& =\left(\tilde{v}_{1} / v_{1}\right)^{m / n} /\left(\tilde{v}_{2} / v_{2}\right)^{l / n}=\left(I_{1}^{m} / I_{2}^{l}\right)^{1 /(l+m)},
\end{aligned}
$$

и неравенство (4.2) установлено. Если же в (4.2) реализуется равенство, то оно будет в правом из неравенств (3.6), а значит, всюду в (3.7). Отсюда следует остальная часть утверждения теоремы.

Таким образом, реализация равенства в (4.2) возможна лишь для таких диффеоморфизмов, которые реализуют равенство в (3.6) (в одном из неравенств). Такие диффеоморфизмы будем называть экстремальными. Их структура, как видно из теоремы 4.1, наиболее близка к структуре конформных отображений. При этом условия (a), (b), (c) вытекают из (3.6) не только для прямых произведений.

Пусть теперь в условиях теоремы 4.1 растяжения $\lambda_{1}, \ldots, \lambda_{l}$ для $g_{1}$ в любой точке $x_{1} \in M_{1}$ не меньше растяжений $\lambda_{l+1}, \ldots, \lambda_{l+m}$ в любой точке $x_{2} \in$ $\in M_{2}$ для $g_{2}$. Тогда диффеоморфизм, задаваемый формулой

$$
g\left(x_{1} ; x_{2}\right)=\left(g_{1}\left(x_{1}\right) ; g_{2}\left(x_{2}\right)\right),
$$

будет экстремальным в классе $\mathfrak{M}$. В самом деле,

$$
K_{n-l}(g)=\underset{x \in M}{\operatorname{essup}} \frac{\left(\lambda_{1} \cdot \ldots \cdot \lambda_{l+m}\right)^{m /(l+m)}}{\lambda_{l+1} \cdot \ldots \cdot \lambda_{l+m}}=\left(I_{1}^{m} / I_{2}^{l}\right)^{1 /(l+m)} .
$$

Рассмотрим несколько частньх случаев.

1. $M_{1}$ - отрезок $[0, a], M_{2}$ - отрезок $[0, b]$,

$N_{1}$ - отрезок $\left[0, a_{1}\right], N_{2}$ - отрезок $\left[0, b_{1}\right]$.
В этом случае $l=1, m=1$, совокупность $\mathfrak{M}$ состоит из всех диффеоморфизмов прямоугольника $M_{1} \times M_{2}$ на прямоугольник $N_{1} \times N_{2}$, переводяших вершины $(0 ; 0),(a ; 0),(0 ; b)$ иІ $(a ; b)$, соответственноо, в вершины $(0 ; 0),\left(a_{1} ;\right.$ $0),\left(0 ; b_{1}\right)$ и $\left(a_{1} ; b_{1}\right)$. Неравенство (4.2) принимает вид

$$
K_{1}(f) \geqslant\left(\frac{a_{1}}{a} \cdot \frac{b}{b_{1}}\right)^{1 / 2}
$$

причем, если $a_{1} / a \geqslant b_{1} / b$, то (4.4) будет аффинным отображением, реализующим равенство, а из теоремы 4.1 нетрудно вывести единственность этого экстремального отображкения (известный результат Греча, см. [4]).

2. $M_{1}$ - область на плоскости $R^{2}, M_{2}$ - интервал $(0, h)$,

$N_{1}$ - область на плоскости $R^{2}, N_{2}$ - интервал $(0, H)$.

Пусть существует аффинное отображение $M_{1}$ на $N_{1}$ с растяжениями $\lambda_{1}$ и $\lambda_{2}$, причем $\lambda_{1}, \lambda_{2} \geqslant H / h$. Тогда в классе диффеоморфизмов $\mathfrak{M}$, переводящих основания цилиндра $M_{1} \times(0 ; h)$ в основания цилиндра $N_{1} \times(0 ; H)$, аффинное отображение будет экстремальным, а для любого отображения $f$ из этого класса, полагая в (4.2) $l=2 ; n=3$, получим

$$
K_{1}(f) \geqslant\left[\frac{\lambda_{1} \lambda_{2}}{(H / h)^{2}}\right]^{1 / 3}
$$

(см. работу автора [6]). Из теоремы 4.1 вытекает также, что экстремальное отображение обязательно имеет в этом случае вид (4.4), где $g_{1}: M_{1} \rightarrow N_{1}$ имеет постоянный якобиан, а $g_{2}$ - линейное.

3. В обозначениях примера 1 раздела 3 пусть $M=Q_{\alpha} \times(\ln a, \ln b)$, $N=Q_{\beta} \times\left(\ln a_{1}, \ln b_{1}\right)$, причем $\beta \geqslant \alpha, \ln \frac{b_{1}}{a_{1}} / \ln \frac{b}{a} \leqslant 1$. Тогда из (3.10) имеem:

$$
I_{1}=\frac{\sqrt{1+\beta}-1}{\sqrt{1+\alpha}-1} \cdot \frac{\sqrt{1+\alpha}}{\sqrt{1+\beta}}
$$

для отображения $g_{1}: Q_{\alpha} \rightarrow Q_{\beta}$, имеющего постоянньй якобиан. Если для этого отображения растяжения в любой точке $\lambda_{1}, \lambda_{2} \geqslant 1$, то экстремальное отображение в классе $\mathfrak{M}$ имеет вид (4.4), а для любого $f$ из этого класса

$$
K_{2}(f) \geqslant\left[\left(\frac{\sqrt{1+\beta}-1}{\sqrt{1+\alpha}-1}\right)^{2} \cdot \frac{\ln \frac{b}{a}}{\ln \frac{b_{2}}{a_{2}}} \cdot \frac{1+\alpha}{1+\beta}\right]^{1 / 3}
$$

Отсюда с помощью дополнительньх конформньх отображенцй получается тот же результат для областей из примера 1 раздела 3.

4. $M_{1}$ и $N_{1}$ окружности радиуса $1, M_{2}$ - круг радиуса $\frac{1}{2} \ln \frac{a+1}{a-1}$ на плоскости Лобачевского, $N_{2}$ - круг радиуса $\frac{1}{2} \ln \frac{b+1}{b-1}$ на плоскости Лобачевско- 
го, причем $b \geqslant a$. Тогда для отображений из совокупности $\mathfrak{M}$, учитывая WARSAW 1983

формулу для площади круга и теорему 4.1, получим

$$
K_{2}(f) \geqslant\left[\frac{b+\sqrt{b^{2}-1}}{a+\sqrt{a^{2}-1}} \cdot \frac{\sqrt{b^{2}-1}}{\sqrt{a^{2}-1}}\right]^{1 / 3} .
$$

Структура экстремального отображения задается формулой (4.4), где $g_{1}$ тождественное отображение единичной окружности на себя, а $g_{2}$ - отображение неевклидова круга $M_{2}$ на неевклидов круг $N_{2}$ с постоянным якобианом и растяжениями $\lambda_{2}, \lambda_{3} \leqslant 1$. Нетрудно проверить, что такое отображение существует, так что приведенная оценка точная. Тот же результат с помоциьо дополнительньх конформньх отображений получается отсюда для торошдальньх областей евклидова пространства $R^{3}$ (ср. результаты Геринга [3]).

\section{Juтepaтypa}

[1] Ф. Р. Г а н т м а х е р, Теория матрии, Наука, Москва 1966.

[2] F. W. Gehring and J. Väisälä, The coefficients of quasiconformality of domains in space, Acta Math. 114 (1965).

[3] Ф. В. Г е р и н $\mathrm{r}$, Экстремальные отображения торов, Сборн. Некоторые проблемы математики и мехакики, Наука, Москва 1970.

[4] H. Gröts ch, Uber möglichst konforme Abbildungen von schlichten Bereichen, Ber. Verh. sächs. Acad. Lpz. 84 (1932), 114-120.

[5] I. A. Cl a r k s o n, Uniformly convex spaces, Trans. Amer. Math. Soc. 40 (1936), 396-414.

[6] В. В. Кр ив ов, Об экстремальных квазиконформных отображсениях в пространстве, Докл. АН СССР 145 (3) (1962), 516-518.

[7] —, Наилучиие экстремальнье отобраэкения в пространстве, ibid. 155 (1) (1964), 38-40.

[8] —, О структуре диффеоморфизмов с минимальной промежсуточной дилатаиией, ibid. 226 (1) (1976), 40-43.

[9] C. L o e w ner, On the conformal capacity in space, J. Math. Mech. 8 (1959), 411-414.

Presented to the Semester

COMPLEX ANALYSIS

February 15-May 30, 1979

\section{EINE EXTREMALCHARAKTERISIERUNG \\ VON UNTERSCHALLGASSTRÖMUNGEN \\ DURCH QUASIKONFORME ABBILDUNGEN}

\section{REINER KUHNAU}

Sektion Mathematik der Martin-Luther-Universität, Halle-Wittenberg Universitätsplatz 6, 401 Halle an der Saale, DDR

\section{Einleitung}

Betrachtet werden in der $x, y$-Ebene stationäre, reibungs- und wirbelfreie Unterschallströmungen eines kompressiblen Gases ohne Zirkulation. Bekanntlich erfüllen dann das (eindeutige) Potential $u(x, y)$ und die (eindeutig vorausgesetzte) Stromfunktion $v(x, y)$ das System

$$
u_{x}=(\alpha / \varrho) v_{y}, \quad u_{y}=-(\alpha / \varrho) v_{x} .
$$

Dabei bedeutet $\alpha$ eine vorgebbare positive Konstante, $\varrho$ die örtliche Dichte. Es sei der Druck $p$ eine reine Funktion von $\varrho$ mit $d p / d \varrho=c^{2}>0 \quad(c=$ örtliche Schallgeschwindigkeit). $p(\varrho)$ sei analytisch (dies wird allerdings nur in $\S 2 . \mathrm{b}$ benötigt). Der Zusammenhang mit dem Geschwindigkeitsquadrat $V^{2}=u_{x}^{2}+u_{y}^{2}$ sei wie üblich durch

$$
\frac{1}{2} d V^{2}=-d p / \varrho \quad \text { (Bernoullische Gleichung) }
$$

gegeben, so daß $\varrho$ eine Funktion von $V^{2}$ wird. Wir setzen noch

$$
f=-(2 / \alpha)\left(p+\frac{1}{2} \varrho V^{2}\right),
$$

wodurch eine Funktion $f=f(\tau)$ mit $\tau=\alpha / \varrho$ definiert werde. Dabei ist bzw. sei für die $\tau$ eines gewissen Intervalles $\mathfrak{F}$

$$
f^{\prime}(\tau)=(\varrho / \alpha)^{2} V^{2}, \quad f^{\prime \prime}(\tau)=2(\varrho / \alpha)^{3}\left(c^{2}-V^{2}\right)>0,
$$

d.h. im Unterschallbereich, auf den wir uns hier beschränken. Die komplexe Funktion $u+i v$ vermittelt eine quasikonforme Abbildung mit der Jacobischen Determinante

$$
J=(\varrho / \alpha) V^{2}
$$

auf ein Parallelschlitzgebiet bei der Umströmung einer Kontur. Wir wollen diese Abbildung im folgenden im Anschluß an die Schlußbemerkung in [13] durch eine 